\title{
Möglicherweise hilfreich in Krisensituationen
}

Fragestellung: Wie wirken sich durch das Internet vermittelte Informationen zur Suizidprävention auf die Nutzer aus?

Hintergrund: Das Internet wird zunehmend zur Vermittlung gesundheitsbezogener Informationen genutzt. Das betrifft auch die Aufklärung über Suizide und Möglichkeiten zu deren Prävention. Dabei sind die Auswirkungen auf das Suizidrisiko nicht eindeutig geklärt. Möglicherweise könnten zusätzliche Suizide getriggert werden und gefährdete Personen sich konkrete Informationen über Methoden beschaffen. Es gibt aber auch Hinweise auf eine Reduktion des Suizidrisikos, wenn der Fokus auf dem konstruktiven Umgang mit schwierigen Lebenssituationen liegt. Vor diesem Hintergrund wurden die Auswirkungen von zur Suizidprävention entwickelten Internetseiten untersucht.

Methodik: In die Studie wurden 161 Studenten eingeschlossen und randomisiert vier Gruppen zugeteilt. Auf einer vorgegebenen Internetseite sollte 10 Minuten lang nach suizidbezogenen Informationen recherchiert

Till B, Tran US, Voracek M et al. Beneficial and harmful effects of educative suicide prevention websites: randomised controlled trial exploring Papageno v. Werther effects. Br J Psychiatry $2017 ; 211: 109-15$ werden. In drei Interventionsgruppen wurde je eine Webseite mit suizidbezogenen Inhalten vorgelegt, in der Kontrollgruppe eine neutrale Webseite. Unmittelbar vor und nach der Exposition sowie eine Woche später wur- den die Probanden mithilfe standardisierter Fragebögen untersucht. Primärer Outcome war das Ausmaß von Suizidideationen. Sekundäre Outcome-Variablen waren Stimmung, Kenntnisse zur Suizidprävention sowie Einstellungen zu Suizid und Inanspruchnahme professioneller Hilfen in Krisensituationen.

Ergebnisse: Es zeigten sich keine Unterschiede zwischen den drei Interventionsgruppen, sodass diese für die weitere Auswertung zusammengefasst wurden. Sowohl unmittelbar nach Exposition als auch eine Woche später zeigte sich eine signifikante Reduktion der Suizidideationen, wenn diese bei Baseline relativ stark ausgeprägt waren; bei niedrigen Baseline-Scores und in der Kontrollgruppe waren keine Veränderungen nachweisbar. In der Interventionsgruppe kam es unmittelbar nach Exposition zu einer vorübergehenden Stimmungsverschlechterung. Die Kenntnisse über Suizidprävention nahmen in der Interventionsgruppe nach Exposition zu, in der Kontrollgruppe zeigten sich keine Effekte. Die Einstellungen zu Suizid und Inanspruchnahme professioneller Hilfe bei Krisen spiegelten keine signifikanten Effekte. In ergänzenden Interviews wurden die auf den Internetseiten vermittelten Informationen generell als hilfreich für Personen mit Suizidideationen eingeschätzt.

Schlussfolgerungen: Internetseiten mit Aufklärung über Suizid und Hilfsangeboten für Menschen in Krisensituationen scheinen hilfreich zu sein und könnten bei relativ höherer Vulnerabilität suizidpräventive Effekte haben.

\section{- Kommentar von Klaus Mann, Mainz}

\section{Internet als zusätzliche Intervention zur Suizidprävention}

Die Studie konnte positive Effekte der ausgewählten Internetseiten für die Nutzer aufzeigen und abgesehen von einer vorübergehenden Verschlechterung der Stimmung unmittelbar nach Exposition waren keine nachteiligen Auswirkungen festzustellen. Neben einer Zunahme der Kenntnisse zur Suizidprävention ergaben sich Hinweise auf möglicherweise suizidprotektive Effekte bei Probanden mit relativ höherer Vulnerabilität entsprechend höheren Scores für Suizidideationen vor Exposition. Dagegen waren bei Probanden mit relativ niedriger Vulnerabilität diesbezüglich keine Effekte erkennbar.

Bei dem untersuchten Kollektiv handelte es sich um junge, überwiegend weibliche Probanden mit hohem Bildungsstand; als suizidal eingeschätzte Probanden waren von der Studie ausgeschlossen. Wie von den Autoren diskutiert, ist nicht klar, inwieweit sich die Ergebnisse auf die Gesamtbevölkerung verallgemeinern lassen, da ältere und männliche Personen ein erhöhtes Suizidrisiko aufweisen. Vor allem ist nicht bekannt, wie die Auswirkungen auf Nutzer mit psychischen Erkrankungen oder in einer gegenwärtigen Krise mit Suizidalität sind. Eine weitere Frage ist, inwieweit langfristige Effekte durch wiederholte Exposition erzielt werden können.
Trotz der eingeschränkten Aussagekraft sollte die Studie zu weiteren Forschungsaktivitäten auf diesem Gebiet anregen. Um negative Auswirkungen zu verhindern, ist insbesondere zu klären, welche spezifischen Inhalte und welche Designcharakteristika von Internetseiten zu suizidpräventiven Effekten beitragen und über welche psychologischen Mechanismen diese zum Tragen kommen. Möglicherweise stellt das Internet durch Bereitstellung angemessener Informationen eine zusätzliche Hilfe für Patienten in Krisensituationen mit Reduktion des Suizidrisikos dar.

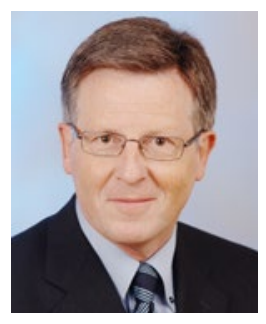

Prof. Dr. med. Dipl.-Ing. Klaus Mann, Mainz

Oberarzt der Klinik für Psychiatrie und Psychotherapie, Universitätsmedizin Mainz E-Mail: Klaus.Mann@unimedizin-mainz.de 